\title{
Temporal contrast control at the PHELIX petawatt laser facility by means of tunable sub-picosecond optical parametric amplification
}

\author{
F. Wagner $\cdot$ C. P. João $\cdot$ J. Fils $\cdot$ T. Gottschall $\cdot$ \\ J. Hein · J. Körner · J. Limpert $\cdot$ M. Roth $\cdot$ \\ T. Stöhlker $\cdot$ V. Bagnoud
}

Received: 30 May 2013/Accepted: 22 October 2013/Published online: 20 November 2013

(C) The Author(s) 2013. This article is published with open access at Springerlink.com

\begin{abstract}
We report on the development of a preamplifier module for temporal contrast enhancement and control at petawatt-class lasers. The module is based on an ultrafast optical parametric amplifier (uOPA), which produces temporally clean pulses at the $60 \mu \mathrm{J}$ level for seeding a chirped pulse amplification (CPA) system, namely the petawatt facility PHELIX. The amplifier module allows for gain reduction in the following amplifiers, resulting in an attenuation of amplified spontaneous emission (ASE) by more than 4 orders of magnitude. Since the ASE of a CPA system linearly depends on the seeding energy, we were able to demonstrate a continuous variation of the temporal contrast by tuning the gain of the uOPA.
\end{abstract}

\footnotetext{
F. Wagner $(\bowtie) \cdot$ M. Roth

Inst. für Kernphysik, Technische Universität Darmstadt,

Schlossgartenstr. 9, 64289 Darmstadt, Germany

e-mail: f.wagner@gsi.de

C. P. João

GoLP, Instituto de Plasmas e Fusao Nuclear-Laboratorio Associado, Instituto Superior Técnico, Av. Rovisco Pais, 1049-001 Lisbon, Portugal

J. Fils · T. Stöhlker · V. Bagnoud

GSI Helmholtzzentrum für Schwerionenforschung $\mathrm{GmbH}$,

Planckstr. 1, 64291 Darmstadt, Germany

T. Gottschall · J. Limpert

Institute of Applied Physics, Friedrich-Schiller-Universität Jena, Albert-Einstein-Str. 15, 07745 Jena, Germany

J. Hein · J. Körner

Inst. für Optik und Quantenelektronik, Friedrich-Schiller-

Universität Jena, Max-Wien-Platz 1, 07743 Jena, Germany

J. Hein · J. Limpert · T. Stöhlker · V. Bagnoud

Helmholtz-Institut Jena, Fröbelstieg 3, 07743 Jena, Germany
}

\section{Introduction}

In the last 10 years, ultra-high-intensity lasers have become widely spread in laboratories around the world. With the ever-increasing achievable on-target intensities, the temporal contrast of such lasers has recently been brought to the foreground of laser development preoccupations. Ultrashort laser pulses are traditionally amplified using the chirped pulse amplification (CPA) technique [1] or optical parametric CPA [2]. Both techniques suffer from an intrinsic temporal pedestal on the nanosecond timescale [3]. In general, the energy of the pulses effectively seeding the amplifier is in the sub-nanojoule range, and the corresponding signal-to-noise ratio after amplification reaches typically $10^{6}-10^{8}$ depending on the seed coupling efficiency. Moreover, since the pulse is stretched during the amplification process, fast optical switches like Pockels cells (PCs) cannot be used to discriminate between the coherent pulse and its pedestal at this stage, while this becomes very complicated after the laser pulses have been re-compressed.

At the same time, the requirements of recent solid target interaction experiments on the temporal contrast become more and more specific. While for several lately proposed experiments like probing ion acceleration mechanisms with thin targets in the sub-micrometer range [4, 5], a high temporal contrast is mandatory to ensure that the target is not destroyed by a prepulse or pedestal; for others, a certain preplasma at the target surface is favorable. Depending on the scale length of the preplasma, different laser absorption mechanisms may dominate resulting in different efficiencies of coupling laser energy into the target. Additionally, ponderomotive and relativistic self-focussing might occur in the preplasma, changing the maximum achievable intensity. Hence, the optimum preplasma strongly depends 
on the application, and an adjustable contrast level is desirable for a user laser facility.

It was demonstrated in 1998 [6] that the amplified spontaneous emission (ASE) in CPA lasers could be suppressed by injection of a clean seed pulse at the microjoule level. By now, a high temporal contrast level is typically achieved by the step-wise approach of temporal pulse cleaning during amplification [7]. In this scheme, the laser pulse is amplified to the millijoule level in a first stage of the laser using a first CPA arrangement and temporally filtered using nonlinear techniques. The resulting pulse has similar properties to the oscillator pulse but, at an energy level many orders of magnitude higher, so that the signalto-noise ratio of the second stage of amplification is much more favorable. One particular implementation of the double CPA concept uses nonlinear cleaning based on crossed wave polarization [8], and it is now widely used in titanium-doped sapphire CPA laser systems around the world. Unfortunately, the double CPA concept turns out to be very unpractical for petawatt-class lasers based on $\mathrm{Nd}$ :glass or Ytterbium-doped amplifiers because the corresponding stretcher and compressor are very bulky, even in the front end of the laser. For this reason, Dorrer et al. [9] proposed an alternate method based on direct amplification of the oscillator pulses in an ultrashort optical parametric amplifier (uOPA). More recently, this method has also been successfully applied to a Ti:sapphire-based CPA system [10] showing its versatility. Because of the instantaneous nature of the parametric process, the signalto-noise ratio of the laser oscillator is not degraded outside of the amplification time window, which can be very short. Ideally, the pump pulse used for the uOPA should be shorter than 1 picosecond to avoid plasma expansion on the picosecond scale. But unfortunately, in previous work [9, 11], the pump pulse was generated by direct amplification of a short pulse in a diode-pumped Nd:YLF laser, and the achievable pulse duration was limited by the bandwidth of the amplification material to more than 5 ps.

In this paper, we demonstrate that the simultaneous operation of an uOPA together with a linear regenerative amplifier allows for continuous tuning of the temporal contrast in a petawatt-class laser. One inherent advantage of our approach is that the pump pulse is generated using a diode-pumped Yb-doped amplifier that has enough bandwidth to support sub-picosecond laser pulses as dictated by solid target interaction experiments. In addition, the requirement on the temporal quality of the pump laser in the uOPA is not as high as for the double CPA scheme, which allows for using a compact and cost-effective stretcher/compressor setup based on a single chirped volume Bragg grating (VBG). Finally, with VBGs available at larger sizes and laser diode-pumped Ytterbium amplifiers nowadays reaching the $1 \mathrm{~J}$ level, the scheme is fully scalable to higher contrast levels. This paves the way for pedestal-free pulses at even higher intensities.

\section{Temporal contrast at the PHELIX laser facility}

The uOPA was developed and optimized to be applied as a pulse cleaner at the PHELIX [12] short-pulse laser system. The system uses the technique of CPA to deliver pulses with minimum duration of $400 \mathrm{fs}$, maximum energy of up to $250 \mathrm{~J}$ at a repetition rate of one shot every $90 \mathrm{~min}$. With a F2-parabolic mirror, the beam can be focused down to reach maximum intensities of up to $2 \times 10^{21} \mathrm{~W} / \mathrm{cm}^{2}$. This requires a good contrast to prevent ionization of targets prior to the main pulse.

In order to understand the contrast deterioration, one has to consider generation and build-up of the high energy pulses in the system. Like in all conventional petawattclass laser systems, the high energy level is achieved by using a multi-stage amplification chain (see Fig. 1). A Ti:sapphire-based short-pulse oscillator (Mira 900, Coherent) provides $100 \mathrm{fs}$ pulses at a nanojoule energy level with a repetition rate of $72 \mathrm{MHz}$. The spectrum of the pulses is shown in Fig. 3a. Even though the laser medium is Ti-sapphire with a maximum cross section around $800 \mathrm{~nm}$, the spectrum is shifted to $1,053 \mathrm{~nm}$ to match the cross section of the subsequent glass amplifiers. Without the contrast-boosting uOPA, these pulses first go to a grating stretcher where they are stretched by a factor of $190 \mathrm{ps} / \mathrm{nm}$ to a duration of $2.4 \mathrm{~ns}$. Then, two successive regenerative $\mathrm{Ti}$-sapphire amplifiers, both working at
Fig. 1 The PHELIX shortpulse laser system

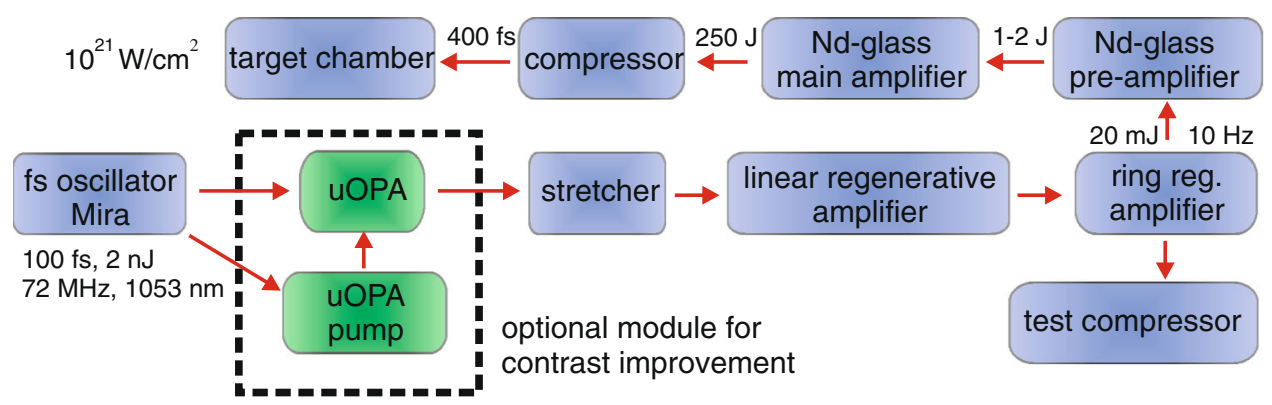




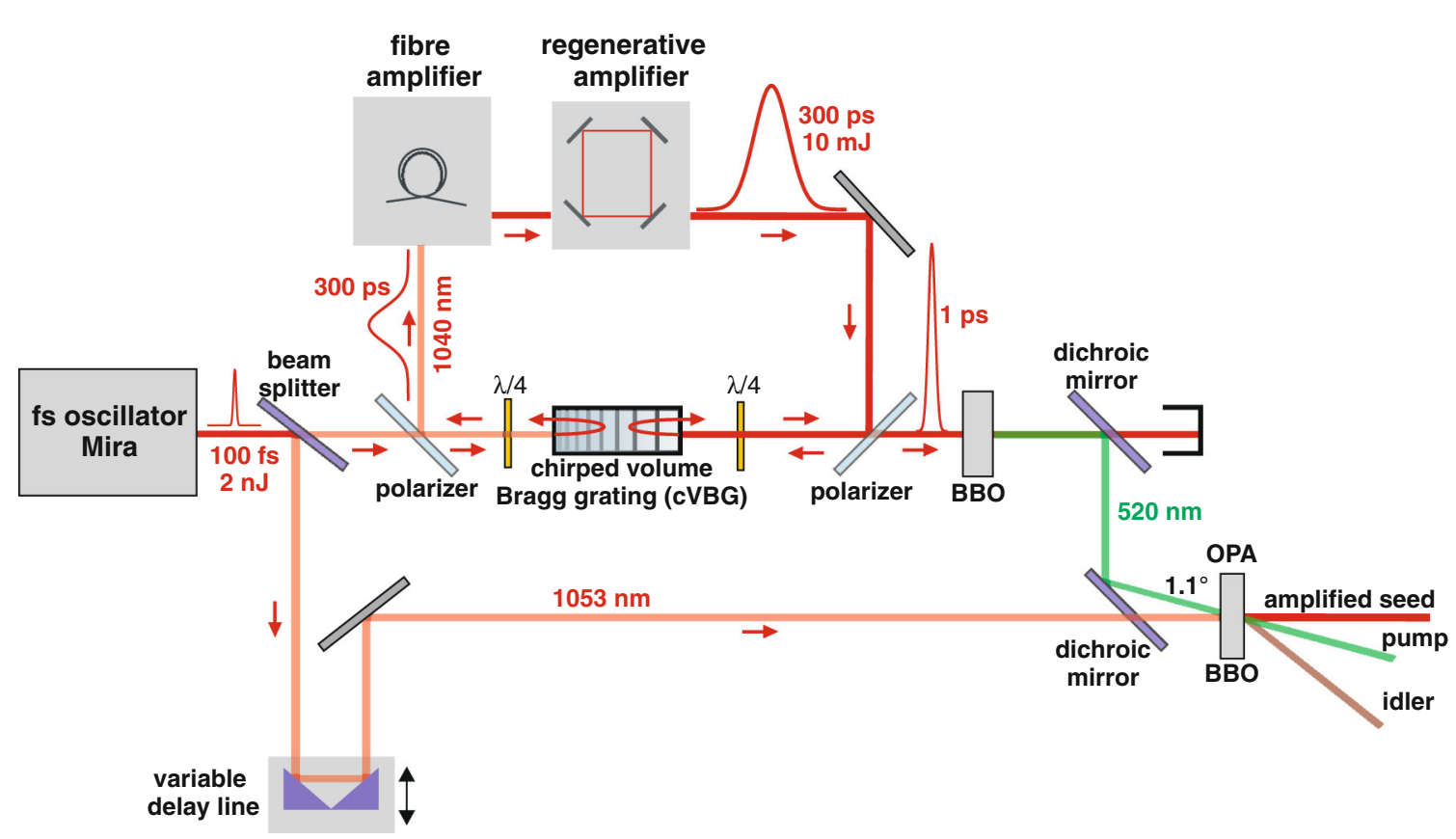

Fig. 2 Setup of the uOPA

$10 \mathrm{~Hz}$, deliver pulses with energies above $20 \mathrm{~mJ}$. One of these pulses is then further amplified up to $250 \mathrm{~J}$ by two $\mathrm{Nd}$ :glass amplifiers and finally recompressed to $400 \mathrm{fs}$ using a grating compressor. In addition, a $30-\mathrm{cm}$ diameter faraday rotator protects the system against back-reflected light, and four isolation stages based on PC pulse pickers are used to eliminate prepulses and the ASE on the nanosecond timescale.

The contrast, in the context of this paper, is defined by the ratio of the main pulse intensity to the intensity of the background radiation of the system. This is linked to the more general problem of signal-to-noise ratio in quantum amplifiers. Here, in the temporal range of some tens of ps up to $1.5 \mathrm{~ns}$ before the pulse, the noise is due to ASE which cannot go below the quantum noise level. Therefore, our approach aims for increasing the signal to improve the contrast. This can be accomplished directly after the shortpulse oscillator.

Other contrast-deteriorative factors, such as spectral clipping, or phase noise in the CPA process, or generation of pre- and postpulses due to multiple reflections, are not addressed by the uOPA and must be considered separately.

\section{Setup of the uOPA}

A main challenge of the uOPA is the generation of a pump pulse which fulfills the following requirements: a duration of not more than one picosecond, as well as a low temporal jitter between the pump and signal pulses. An additional demand for the pump laser is a setup as compact as possible to be suitable for the PHELIX system. The setup of the uOPA is shown in a schematic in Fig. 2. In our realization, both pump and signal pulses originate from the same oscillator in order to account for the low jitter requirements. By using a 50:50 beamsplitter, half of the beam directly propagates to the beta barium borate (BBO) crystal, where it is amplified in the OPA process. A motorized delay line is used to temporally overlap the seed with the pump pulse. The other part is amplified in a compact CPA system and serves as the pump pulse in the OPA. First, the pulse is stretched to $300 \mathrm{ps}$ after being reflected in a VBG (OptiGrate Corp.). This VBG was designed to reflect a 5-nm bandwidth pulse centered at $1,040 \mathrm{~nm}$. The central wavelength matches the amplifiers which both use Ytterbium as active material. The main advantage of the VBG compared with conventional grating-based stretcher-compressor assemblies is its compactness, cost and ease of use. Both stretching and recompression of the pulse are carried out in the same VBG with dimensions in the order of a few centimeters. In Fig. 3a, the part of the spectrum which is extracted from the original spectrum delivered by the fs oscillator is shown. It can clearly be seen (the spectra are not normalized with respect to each other) that the efficiency of the VBG in the dedicated wavelength range is nearly $100 \%$. Besides its compactness, this is another important advantage of the VBG. The stretched pulses are then amplified in two consecutive amplifiers. The first amplification takes place in a two-stage Ytterbium-doped fiber amplifier. In a 

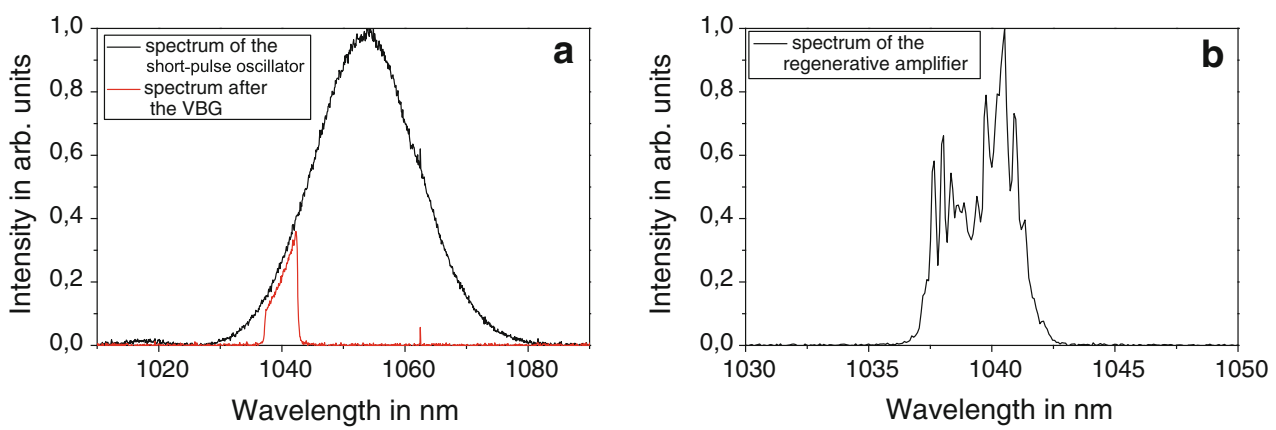

Fig. 3 Spectra of the short-pulse oscillator (a-black), after the VBG (a-red) and output of the regenerative amplifier for a pulse energy of $4.5 \mathrm{~mJ}(\mathbf{b})$

single mode fiber, the stretched pulses with an average power of 1-2 $\mathrm{mW}$ and a repetition rate of $72 \mathrm{MHz}$ are amplified to $160 \mathrm{~mW}$. The repetition rate is then reduced to $1 \mathrm{MHz}$ using an acousto-optical modulator (AOM) synchronized with the PHELIX laser system. A second stage then creates an output of $1 \mathrm{~W}$ corresponding to pulse energies of $1 \mu \mathrm{J}$. The beam then goes to a regenerative ring amplifier operating at $10 \mathrm{~Hz}$ [13]. The amplifier uses $\mathrm{Yb}: \mathrm{KGW}$ as an active medium pumped by a laser diode module (Lastronics $\mathrm{GmbH}$ ) which is capable of delivering up to $2.4 \mathrm{~kW}$ peak power at $940 \mathrm{~nm}$. Output of the ring is a pulse train with nominal pulse energies of $10 \mathrm{~mJ}$ and a bandwidth of $5 \mathrm{~nm}$ centered at $1,040 \mathrm{~nm}$. However, due to nonlinear effects, the spectrum exhibits strong modulations at higher energies. Therefore, we decided to limit the output energy to $4.5 \mathrm{~mJ}$, an energy level for which no visible effect on the parametric amplification could be detected. The spectrum after the ring amplifier (Fig. 3b) exhibits the same bandwidth as the input beam, which enables compression of the pulses to 1 ps with the VBG. This is accomplished by using the VBG in the direction opposite to the stretching direction. It should be noted that the compression factor applied by the VBG does not address the additional dispersion introduced by the propagation through the fiber and material in the regenerative amplifier. For this reason, the output pulse is not as short as its corresponding Fourier transform limit (600 fs), but rather lightly chirped and its duration increased to 1 picosecond.

In order to use the pulses as pump pulses in the OPA process, they need to be frequency doubled, which is accomplished in a BBO crystal. The frequency-doubling stage for the pump laser and the parametric amplifier were simulated and dimensioned using the Miro software [14] with the specific module handling four wave mixing of broadband pulses. The doubling crystal is located about $250 \mathrm{~cm}$ from the waist of the regenerative cavity, and the pump-laser beam diameter equals $2.9 \mathrm{~mm}$ (FWHM) at the crystal location, due to the natural divergence of the beam.
This produces a maximum available intensity of $150 \mathrm{GW} /$ $\mathrm{cm}^{2}$. For this intensity, we estimated that a $2.9 \mathrm{~mm}$ Type I BBO crystal offers the optimal length to drive the frequency conversion into saturation, and consequently, it minimizes the energy fluctuation noise. A fraction of $50 \%$ from the $4.5 \mathrm{~mJ}$ at fundamental wavelength can be converted to green, which results in a pump-pulse energy of about $2.3 \mathrm{~mJ}$ at the OPA-crystal. Two dichroic mirrors are used to separate the pump beam at $520 \mathrm{~nm}$ from the residual infrared radiation that has not been converted in the BBO crystal. The second dichroic mirror also serves as a beam combiner for the signal and pump, since it is highly transmissive for infrared and highly reflective for $520 \mathrm{~nm}$. To meet the requirements for phase matching in the BBO crystal, the angle between the pump pulse at $520 \mathrm{~nm}$ and the seed pulse at $1,053 \mathrm{~nm}$ has to be unequal to zero degrees. Using the Miro software, an optimum angle of 1.1 degrees was calculated. This also enables to separate the amplified signal from the residual pump and the idler.

Due to the long optical path in the fiber amplifier as well as the regenerative amplifier, a single pulse generated by the oscillator cannot be used simultaneously as pump and seed pulse in the OPA. Therefore, two different pulses with a temporal separation of 1.2 microseconds are used in our setup which was identified as a possible source of jitter or drift.

First, jitter was minimized in our setup by locking the oscillator to an external $72 \mathrm{MHz}$ reference rf signal which is stabilized to the $\mathrm{Hz}$ level. This ensures the stability between the subsequent pulses of the oscillator. Second, a compact design allows for minimizing the impact of local vibration effects.

With a pump-pulse energy of $2.3 \mathrm{~mJ}$, we reach saturation in the OPA, which corresponds to a conversion efficiency of about $3 \%$. This value can be explained by the not optimally matched pulse duration of the pump and seed pulses. To improve the conversion efficiency, the seed pulse was stretched to a duration of $300 \mathrm{fs}$ using multiple reflections from two chirped mirrors. Consequently, we were able to double the efficiency. However, the outcome 
of this was fluctuations in the pulse duration after re-compression of the amplified pulses after the regenerative amplifiers of the PHELIX front end (Fig. 1). This was attributed to the jitter between the seed and the pump pulses resulting in a nonlinear, pulse-to-pulse varying spectral phase which cannot be compensated for within the compressor. Therefore, we decided to operate the uOPA at the lower conversion efficiency in favor of a better longterm stability of the whole system.

In order to maintain a constant energy output level of the OPA, the temporal overlap between the pump and seed pulses needs to be preserved during operation. In the course of the day, we observed an unidirectional drift of a few ps between both pulses which was related to the warm-up of the regenerative cavity resulting in a longer optical path for the pump pulse. This effect can be compensated for by adjusting the variable delay line for the signal beam. This is achieved by an automated closed-loop locking the delay line position to the uOPA output energy. When this loop is active, the synchronization is maintained in routing operation over more than $12 \mathrm{~h}$ per day. As far as jitter is concerned, with the compressed signal pulse, we could not observe any instability that could be attributed to it. With the pump-pulse duration being by a factor of ten longer compared with the signal pulse, the low jitter requirement becomes less critical.

\section{Integration to the PHELIX short-pulse laser chain and contrast measurements}

The uOPA module was integrated to the PHELIX shortpulse system and can be used at any time during daily operation. Switching between the configuration with and without the pulse cleaner is very easy and can be arranged within minutes. As illustrated in Fig. 1, the uOPA is placed directly behind the short-pulse oscillator. After the uOPA the amplified signal pulse is sent to the grating stretcher and subsequently to the two regenerative amplifiers of the PHELIX short-pulse front end. Due to the higher input signal the amplification in the regenerative amplifiers can be reduced to deliver the same output energy level of $20 \mathrm{~mJ}$. This is accomplished by decreasing the pumping laser energy in the linear regenerative amplifier which results in less ASE production. Because the optical path and timing of the regenerative amplifier is identical for both operation modes, the changeover between the two configurations is straightforward.

To measure the contrast with the new module, the laser pulses amplified in the front end were sent to a local compressor (Fig. 1). Because the amplification in the glass amplifier is not saturated, we do not expect a degradation of the temporal contrast between the front part of the pulse and its peak. In addition, the ASE contribution of the glass

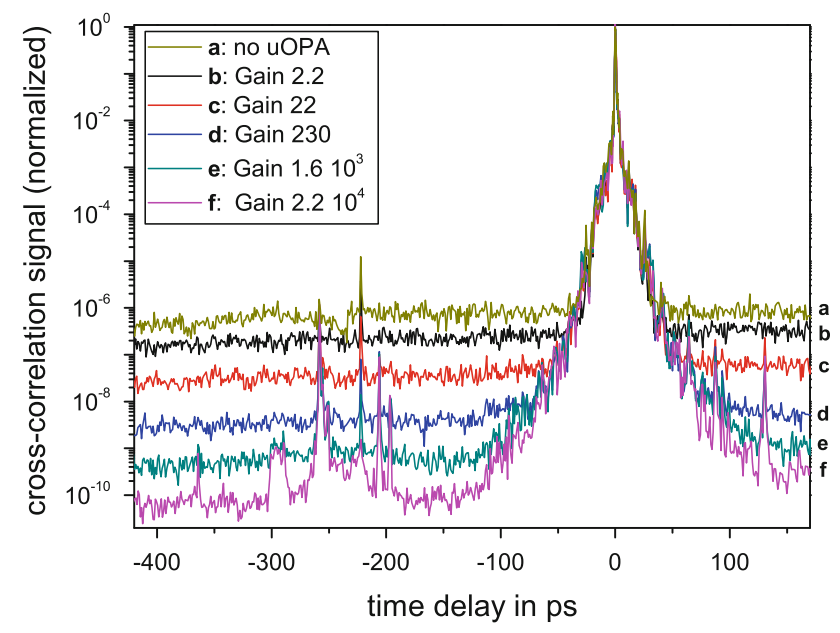

Fig. 4 Improvement of the ASE contrast for different gain levels in the uOPA

amplifier was estimated theoretically and measured experimentally to be negligible to play any role in the picosecond ASE contrast level of the laser pulse. Therefore, we believe that the measurement done at the output of the front end is also representative of the temporal contrast of the laser pulse at full energy. At this stage, the system is still operating at $10 \mathrm{~Hz}$. Hence, a scanning third-order cross-correlator with high dynamic range (Sequoia, Amplitude Technology) can be used. We performed the measurement for different gain levels in the uOPA by variation of the OPA pump-pulse energy. This was done with a half-waveplate and polarizer arrangement, used together with a photodiode that monitored the uOPA gain on an oscilloscope. For each gain level, the subsequent regenerative amplifier was maintained in saturation by adjusting the pump energy of the driving Nd:YAG pump laser using a waveplate and polarizer. By doing so, the energy at the output of the regenerative amplifier remains nearly unchanged, while the gain is shifted from the regenerative amplifier to the uOPA and the ASE originating in the regenerative amplifier accordingly reduced. The results are shown in Fig. 4. In order to compare the different ASE levels, the curves were normalized with respect to each other. This was carried out by overlapping the slopes of each curve. The upper curve was recorded with no gain in the OPA corresponding to the standard setup without the uOPA module. The ASE is at the $10^{-6}$ level which is typical for a front end with no additional contrastimproving components. Starting with a gain factor of 2.2 and increasing it stepwise by factors of nearly 10, the ASE drops down almost linearly with the OPA gain. For a gain of $2.2 \times 10^{4}$, the ASE level exceeds the detection limit of the cross-correlator which is slightly below $10^{-10}$.

The curve with the highest contrast exhibits a slowly rising slope ranging up to $100 \mathrm{ps}$ before the peak. This is 


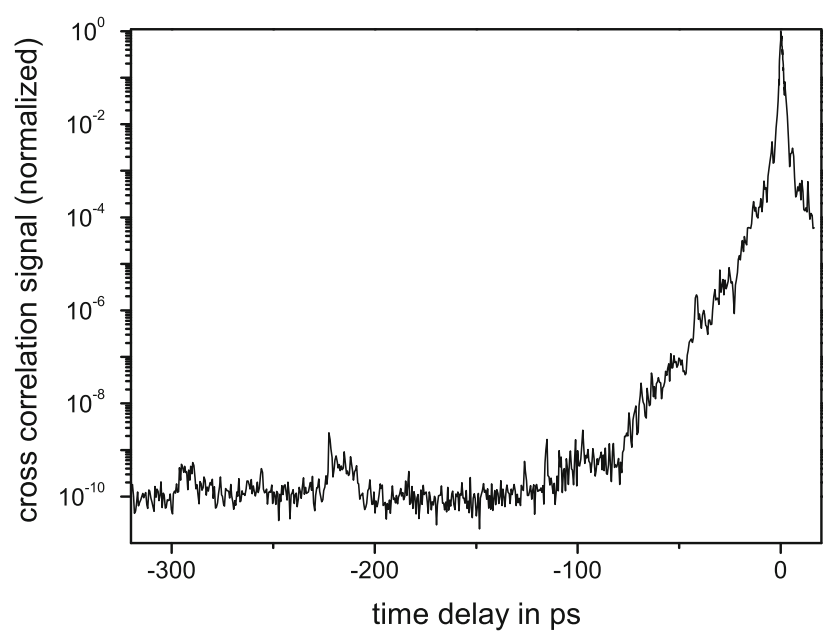

Fig. 5 Temporal contrast after the removal of all prepulses for a gain of $2 \times 10^{4}$ in the uOPA

attributable to coherent effects such as clipping of the spectrum or scattering from the diffraction gratings in the stretcher [15]. This slope can be detrimental to the experiments that are the most sensitive to pre-ionization effects. Therefore, solutions like increasing the stretcher bandwidth by $50 \%$ and replacing the gratings for higher quality components are currently considered.

In addition, when a very high ASE contrast is achieved, prepulses between -300 and $-200 \mathrm{ps}$ become visible. These effects are not addressed by the uOPA and therefore have to be treated separately. In general, prepulses can be generated in a CPA system from the interplay between postpulses and nonlinear effects (nonlinear Kerr effect) [16]. In particular, we could identify that the prepule at 200 ps was due to double reflections from a PC with parallel surfaces in the PHELIX front end. Hence, it was not addressed by the uOPA. By switching to a specially designed PC with tilted end faces, the prepulse could be removed. Furthermore, the prepulses around 250 ps which were caused by double reflections from several cube polarizers in the front end could be eliminated by exchanging these polarizers.

The prepulse at about 220 ps is caused by injection of another pulse from the oscillator into the regenerative amplifiers. Therefore, it drops down with increasing uOPA gain, as well. The final contrast level after the prepulse removal and with an applied gain of $2 \times 10^{4}$ in the uOPA is shown in Fig. 5.

\section{Conclusion}

A compact preamplifier based on sub-picosecond OPA was developed and successfully integrated to the PHELIX petawatt laser system. With this module, an improvement of the ASE contrast from $10^{6}$, which is typical for CPA systems, to more than $10^{10}$, could be demonstrated. This enlarges the spectrum of experiments feasible at the PHELIX laser facility. Preliminary tests on ion acceleration with thin targets showed that the current contrast allows studying laser matter interaction with targets in the submicrometer range. In particular, this enables testing new laser ion acceleration mechanisms like the laser break-out afterburner [4], or radiation pressure acceleration [5] at PHELIX which are planned for the near future.

Our measurement also confirms that the ASE level in a CPA system linearly depends on the energy of the seed pulse. Consequently, by proper tuning, the gain between the uOPA and the following linear regenerative amplifier, an arbitrary ASE level between 6 and 10 orders of magnitude can be accomplished, while maintaining the total output energy level of the system. This is very favorable for a user laser facility, since a controllable preplasma can be useful for many types of applications.

Acknowledgments This work was supported by the BMBF 06DA90441 and the Helmholtz-Institute Jena.

Open Access This article is distributed under the terms of the Creative Commons Attribution License which permits any use, distribution, and reproduction in any medium, provided the original author(s) and the source are credited.

\section{References}

1. D. Strickland, G. Mourou, Opt. Commun. 55, 447-449 (1985)

2. A. Dubietis, G. Jonusauskas, A. Piskarskas, Opt. Commun. 88, 437-440 (1992)

3. V.V. Ivanov, A. Maksimchuk, G. Mourou, Appl. Opt. 42, 7231-7234 (2003)

4. L. Yin, B.J. Albright, B.M. Hegelich, J.C. Fernández, Laser Part. Beams 24, 291-298 (2006)

5. A. Macchi, S. Veghini, T. Liseykina, F. Pegoraro, New Phys. 12, 045013 (2010)

6. J. Itatani, J. Faure, M. Nantel, G. Mourou, S. Watanabe, Opt. Commun. 148, 70-74 (1998)

7. M.P. Kalashnikov, E. Risse, H. Schönnagel, W. Sandner, Opt. Lett. 30, 923-925 (2005)

8. A. Jullien, O. Albert, F. Burgy, G. Hamoniaux, J.-P. Rousseau, J.-P. Chambaret, F. Augé-Rochereau, G. Chériaux, J. Etchepare, N. Minkovski, S.M. Saltiel, Opt. Lett. 30, 920-922 (2005)

9. C. Dorrer, I.A. Begishev, A.V. Okishev, J.D. Zuegel, Opt. Lett. 32, 2143-2145 (2007)

10. C. Liu, Z. Wang, W. Li, Q. Zhang, H. Han, H. Teng, Z. Wei, Opt. Lett. 35, 3096-3098 (2010)

11. I. Musgrave, W. Shaikh, M. Galimberti, A. Boyle, C. HernandezGomez, K. Lancaster, R. Heathcote, Appl. Opt. 49, 6558-6562 (2010)

12. V. Bagnoud, B. Aurand, A. Blazevic, S. Borneis, C. Bruske, B. Ecker, U. Eisenbarth, J. Fils, A. Frank, E. Gaul, S. Goette, C. Haefner, T. Hahn, K. Harres, H.-M. Heuck, D. Hochhaus, D.H.H. Hoffmann, D. Javorková, H.-J. Kluge, T. Kuehl, S. Kunzer, M. Kreutz, T. Merz-Mantwill, P. Neumayer, E. Onkels, D. Reemts, 
O. Rosmej, M. Roth, T. Stoehlker, A. Tauschwitz, B. Zielbauer, D. Zimmer, K. Witte, Appl. Phys. B 100, 137-150 (2010)

13. C. P. João, J. Körner, M. Kahle, H. Liebetrau, R. Seifert, M. Lenski, S. Pastrik, J. Hein, T. Gottschall, J. Limpert, G. Figueira and V. Bagnoud, High-power Yb:KYW picosecond regenerative amplifier for optical parametric amplifier pumping. Proc. SPIE 8001, International Conference on Applications of Optics and Photonics, 80011E (July, 2011)
14. O. Morice, Opt. Eng. 42, 1530-1541 (2003)

15. C. Hooker, Y. Tang, O. Chekhlov, J. Collier, E. Divall, K. Ertel, S. Hawkes, B. Parry, P.P. Rajeev, Opt. Exp. 19, 2193-2203 (2011)

16. N.V. Didenko, A.V. Konyashchenko, A.P. Lutsenko, S.Yu. Tenyakov, Opt. Exp. 16, 3178-3190 (2008) 